\title{
Optimal pulses for arbitrary dispersive media
}

\author{
M. A. Alonso \\ alonso@optics.rochester.edu
}

\section{T. Setälä}

\section{A. T. Friberg}

\author{
Department of Applied Physics, Aalto University, P. O. Box 13500, FI-00076 Aalto, Finland \\ The Institute of Optics, University of Rochester, Rochester, NY 14627, USA \\ Department of Applied Physics, Aalto University, P. O. Box 13500, FI-00076 Aalto, Finland
}

A variational procedure is given for finding the pulses for which the initial temporal rms width and the rate of increase of this width are jointly minimized for propagation in non-absorbing media with arbitrary dispersive properties. We show that, while in linearly dispersive media the optimal pulses are Gaussian, in other situations such as a hollow metallic waveguide or for purely cubic dispersion departures from Gaussian behavior become evident. An interpretation of the results in terms of suitable phase-space representations is also given. [DOI: 10.2971/jeos.2011.11035]

Keywords: Light pulses, pulse quality factor, phase space, dispersion, variational methods

\section{INTRODUCTION}

Continuous-wave laser beams are traditionally characterized by the so-called $M^{2}$ parameter, which is a phase-space quality factor related to the product of the root-mean-square (rms) beam waist width and the rms width of the far-field diffraction angle [1]. For one-dimensional fields, the square of the transverse rms intensity width obeys in paraxial free-space propagation a quadratic (parabolic) law as a function of distance $z$ from the waist [2]. The parameter $M^{2}$, however, is preserved under paraxial free propagation and also in first-order (ABCD) optical systems [3]. Since a Gaussian function attains the minimum product of the widths, it is used to normalize this quality factor, so that $M^{2}=1$ is attained only for Gaussian beams and $M^{2} \geq 1$ in general [4,5]. Apertured fields, nonparaxial propagation, and material media lead to further refinements of the optimal phase-space theory. For long pulses a time-dependent $M^{2}$ factor can be used. However, for ultrashort pulses in the few-cycle range the spectrum influences $M^{2}$ and the optimum beam in terms of high directionality and small spot size no longer has a Gaussian profile [6].

A well-known space-time analogy exists between spatial paraxial propagation of stationary optical fields and temporal evolution of plane-wave pulses in linearly dispersive homogeneous media [7]. The analogy is manifested by the fact that in slowly-varying approximations, the underlying dynamics in both cases are governed by similar, Schrödinger-type equations [8, 9]. Hence, a pulse quality factor $M_{\tau}^{2} \geq 1$ can be introduced as a time-bandwidth product in terms of the second moments of intensity and spectral density [10]. In first-order temporal $\mathrm{ABCD}$ systems $M_{\tau}^{2}$ remains constant and assumes its minimum value for Gaussian pulses. The temporal evolution of general (non-Gaussian) pulses in such dispersive elements is fully analogous with the spatial counterpart [3,10]. Experiments with short pulses down to the femtosecond regime have confirmed the validity of the pulse quality factor description for plane-wave pulses in linearly dispersive media [11].

In this work we consider the one-dimensional propagation of pulses (such as plane waves or guided modes) in homogeneous transparent media of arbitrary dispersive properties. Rather than focusing on a product of the pulse length and spectral width in some input location, as in the pulse quality factor, we look for pulses that minimize the product of the pulse durations in the initial plane and after propagating over a long distance. The optimal pulses depend on the dispersive characteristics of the medium and are not, in general, of Gaussian spectrum. We employ a general variational method based on Lagrange multipliers for finding the optimal pulse profiles in any non-absorbing medium and analyze, as examples, media with specific dispersion relations. The results are illustrated in terms of modified Wigner functions that describe pulse propagation in transparent media with any dispersive properties exactly as phase-space shearing [12].

\section{PULSE PROPAGATION IN DISPERSIVE MEDIA}

The forward-propagating solutions to the wave equation in a one-dimensional dispersive medium can be written as super- 
positions of monochromatic components

$$
U(z, t)=\frac{1}{\sqrt{2 \pi}} \int A(\omega) \exp \{\mathrm{i}[k(\omega) z-\omega t]\} \mathrm{d} \omega,
$$

where $A(\omega)$ is the field's spectral amplitude and $k(\omega)=\omega n(\omega) / c$ is the wavenumber corresponding to the frequency $\omega$, with $n$ being the frequency-dependent refractive index and $c$ the speed of light in vacuum. Let us assume for now that the medium's absorption is negligible, so that $k$ is real. The range of integration in Eq. (1) then is a window of frequencies in which this assumption is valid. (Some comments on the effects of absorption in the results obtained here are given in the concluding remarks.) For simplicity, we use the analytic signal representation of the field, meaning that the integral involves only positive frequencies, and $U$ is a complex function whose real part represents the true physical pulse.

The total power contained in the pulse is proportional to the following quantity

$$
\phi=\int|U(z, t)|^{2} \mathrm{~d} t=\int|A(\omega)|^{2} \mathrm{~d} \omega,
$$

where the integral in the first equality is taken over all time. Similarly, the temporal moments as functions of distance can be defined as

$$
\overline{t^{m}}(z)=\frac{1}{\phi} \int|U(z, t)|^{2} t^{m} \mathrm{~d} t .
$$

Let us define the shorthand notation

$$
\kappa_{l m n}=\frac{(-\mathrm{i})^{l}}{\phi} \int\left[k^{(m)}(\omega)\right]^{n} A^{*}(\omega) A^{(l)}(\omega) \mathrm{d} \omega,
$$

where a superindex $(j)$ denotes the $j$ th derivative. The first and second moments can then be found through the substitution of Eq. (1) into Eq. (3) to be given by

$$
\begin{aligned}
\bar{t}(z) & =\kappa_{100}+z \kappa_{011}, \\
\overline{t^{2}}(z) & =\kappa_{200}+z\left(2 \kappa_{111}-\mathrm{i} \kappa_{021}\right)+z^{2} \kappa_{012} \\
& =\kappa_{200}+z 2 \Re\left(\kappa_{111}\right)+z^{2} \kappa_{012} .
\end{aligned}
$$

Note that all $\kappa_{l m n}$ in the above equations are real, with the exception of $\kappa_{111}$. However, it can be shown through integration by parts that $2 \Im\left(\kappa_{111}\right)=\kappa_{021}$. This relation was used in the last step in Eq. (6).

The pulse's temporal width as a function of distance can be characterized by its root-mean-square (rms) width, which is given by

$$
\begin{aligned}
& \Delta t(z)=\sqrt{\overline{t^{2}}(z)-\bar{t}^{2}(z)} \\
& =\sqrt{\kappa_{200}-\kappa_{100}^{2}+z 2\left[\Re\left(\kappa_{111}\right)-\kappa_{100} \kappa_{011}\right]+z^{2}\left(\kappa_{012}-\kappa_{011}^{2}\right)} .
\end{aligned}
$$

The temporal width of the pulse at the initial position $(z=0)$ then is given by

$$
\Delta t_{0}=\Delta t(0)=\sqrt{\kappa_{200}-\kappa_{100}^{2}} .
$$

On the other hand, the rate at which the temporal width increases after propagating long distances is given by

$$
\Delta s_{\infty}=\lim _{z \rightarrow \infty} \frac{\Delta t(z)}{z}=\sqrt{\kappa_{012}-\kappa_{011}^{2}} .
$$

Notice that $\Delta s_{\infty}$ has units of inverse velocity.

These two measures are analogous to the initial transverse spatial width and the far-field directional spread of a stationary beam.

\section{OPTIMAL PULSES}

We now search for the fundamental lower bounds for the measures $\Delta t_{0}$ and $\Delta s_{\infty}$. For this purpose, we employ a variational approach in order to find the pulses that jointly minimize both measures. Mathematically, this is achieved by requiring that a positive linear combination of these measures (or, equivalently, their squares) be stationary under infinitesimal variations of the spectral amplitude. Note that the spectral amplitude and its complex conjugate can be treated as independent degrees of freedom. The condition for stationarity then takes the form

$$
\frac{\delta \Delta t_{0}^{2}}{\delta A^{*}}+\mu^{2} \frac{\delta \Delta s_{\infty}^{2}}{\delta A^{*}}=0,
$$

where $\mu^{2}$ is a positive quantity (a Lagrange multiplier). For computational purposes it is convenient to parameterize the solution not in terms of $\Delta t_{0}$ or $\Delta s_{\infty}$ but in terms of $\mu$, so that both $\Delta t_{0}$ and $\Delta s_{\infty}$ for the optimal pulses depend on this parameter. In other words, in a plane whose axes are $\Delta t_{0}$ and $\Delta s_{\infty}$, the widths obtained through this variational procedure describe a curve (where each point is calculated with a different value of $\mu$ ). This curve corresponds to the boundary between the allowed and forbidden pairs of these width measures for any pulse.

By using the fact that

$$
\frac{\delta \kappa_{l m n}}{\delta A^{*}}=\frac{(-\mathrm{i})^{l}\left[k^{(m)}(\omega)\right]^{n} A^{(l)}(\omega)-\kappa_{l m n} A(\omega)}{\phi},
$$

(where the last term results from the functional derivative of $\phi$ in the denominator) the substitution of Eqs. (8) and (9) into Eq. (10) and the use of the chain rule lead to the following equation:

$$
\begin{aligned}
A^{\prime \prime}(\omega) & -2 \mathrm{i} \kappa_{100} A^{\prime}(\omega)+\left[\left(\kappa_{200}-2 \kappa_{100}^{2}\right)+\mu^{2}\right. \\
& \left.\times\left\{\kappa_{012}-\kappa_{011}^{2}-\left[k^{\prime}(\omega)-\kappa_{011}\right]^{2}\right\}\right] A(\omega)=0 .
\end{aligned}
$$

(The correctness of this equation can be verified by multiplying it by $A^{*}$ and integrating over all frequencies.) This equation can be simplified by factoring out a linear phase as $A(\omega)=\bar{A}(\omega) \exp \left(\mathrm{i} \kappa_{100} \omega\right)$, leading to

$$
\bar{A}^{\prime \prime}(\omega)+\left(\Delta t_{0}^{2}+\mu^{2}\left\{\Delta s_{\infty}^{2}-\left[k^{\prime}(\omega)-\kappa_{011}\right]^{2}\right\}\right) \bar{A}(\omega)=0 .
$$

To solve this equation, we can write it as an eigenvalue system,

$$
-\bar{A}^{\prime \prime}(\omega)+\mu^{2}\left[k^{\prime}(\omega)-\kappa_{011}\right]^{2} \bar{A}(\omega)=\Lambda \bar{A}(\omega)
$$

where the eigenvalue $\Lambda$ equals $\Delta t_{0}^{2}+\mu^{2} \Delta s_{\infty}^{2}$. Notice that this equation is mathematically analogous to a onedimensional time-independent Schrödinger equation, in which $\mu^{2}\left[k^{\prime}(\omega)-\kappa_{011}\right]^{2}$ plays the role of the potential, and $\Lambda$ corresponds to the energy. 
The procedure to find the lower bounds for the spread measures is as follows: First, one chooses the constant $\kappa_{011}$ to coincide with $k^{\prime}\left(\omega_{0}\right)$, so that the "potential" $\mu^{2}\left[k^{\prime}(\omega)-\kappa_{011}\right]^{2}$ has a minimum at $\omega_{0}$, which then is approximately the central frequency of the resulting optimal pulse. The next step is to solve the eigenvalue problem for a given value of $\mu$ and select the "ground state" solution, corresponding to the smallest $\Lambda$. Given the dip-like shape of the potential around $\omega_{0}$, this ground-state solution can be expected to be bound, i.e. to decay away from $\omega_{0}$. Finally, by using the resulting spectral amplitude, one calculates $\Delta t_{0}$ and $\Delta s_{\infty}$, which for the optimal pulses are functions of $\mu$, and are denoted by a superindex "opt". [The correctness of the results can be verified by checking that $\Lambda=\Delta t_{0}^{\mathrm{opt}^{2}}(\mu)+\mu^{2} \Delta s_{\infty}^{\mathrm{opt}^{2}}(\mu)$ is satisfied.] This procedure must be carried out for each value of $\mu^{2}$.

\section{PHASE-SPACE INTERPRETATION}

It is well known that the Wigner function gives an illustrative representation of paraxial diffraction, as well as of the propagation of pulses in the case of linear dispersion [13, 14]. A modified version of the Wigner function was proposed recently that describes the propagation of a pulse through a transparent medium with arbitrary dispersion exactly in terms of a distribution $B_{v}(z, t, v)$ that resembles a statistical mechanical distribution of particles traveling at different velocities $v$ [12]. The two key properties of this distribution are: i) The intensity is given by its integral over velocity:

$$
\int B_{v}(z, t, v) \mathrm{d} v=|U(z, t)|^{2},
$$

and ii) this distribution is constant along trajectories of constant velocity:

$$
B_{v}(z, t, v)=B_{v}\left(0, t-\frac{z}{v}, v\right)
$$

The definition of $B_{v}(z, t, v)$ depends on the dispersion relation, according to a geometric prescription given in Ref. [12]. In this work, it is more useful to write this representation in terms of the inverse of the velocity, $s=1 / v$, so that the phase space distribution is

$$
B_{s}(z, t, s)=s^{2} B_{v}\left(z, t, s^{-1}\right),
$$

and the properties above become

$$
\begin{aligned}
\int B_{s}(z, t, s) \mathrm{d} s & =|U(z, t)|^{2}, \\
B_{s}(z, t, s) & =B_{s}(0, t-s z, s) .
\end{aligned}
$$

In terms of this definition, the two spreads defined earlier are given by

$$
\begin{aligned}
\Delta t_{0}^{2} & =\frac{\iint t^{2} B_{s}(0, t, s) \mathrm{d} s \mathrm{~d} t}{\iint B_{s}(0, t, s) \mathrm{d} s \mathrm{~d} t}, \\
\Delta s_{\infty}^{2} & =\frac{\iint s^{2} B_{s}(0, t, s) \mathrm{d} s \mathrm{~d} t}{\iint B_{s}(0, t, s) \mathrm{d} s \mathrm{~d} t} .
\end{aligned}
$$

Hence, the two measures are the rms widths of this distribution for $z=0$ in the $t, s$ phase space. For the case of linear dispersion, this distribution reduces to the standard Wigner function, and for a Gaussian pulse, this representation reduces to a Gaussian in the two phase-space variables $t, s$ for $z=0$, which is the most jointly localized Wigner distribution possible. For more complicated dispersion relations, $B_{S}$ is no longer a Gaussian for Gaussian pulses, and in fact, no pulse exists in general that makes this distribution Gaussian. However, the optimal pulses described here make these distributions as localized in phase space as possible.

\section{EXAMPLES}

The ground-state solutions to Eq. (14) are known analytically only for a few cases. In general, it is necessary to apply a numerical method (such as the one described in Appendix A).

\subsection{Linear dispersion}

The simplest example results from assuming that the dispersion is linear over the spectral extent of the pulse, that is, the wavenumber is given by

$$
k(\omega)=\beta_{0}+\beta_{1}\left(\omega-\omega_{0}\right)+\beta_{2} \frac{\left(\omega-\omega_{0}\right)^{2}}{2},
$$

where $\omega_{0}$ is the central frequency of the pulse. Of course, in reality, this is an approximation, valid only for pulses with sufficiently narrow spectral widths. However, this case is important because it leads to a full mathematical analogy with the problem of paraxial free-space diffraction $[3,9]$. Therefore, the results of this method should lead to optimal pulses with Gaussian spectrum [10]. By choosing $\kappa_{011}=\beta_{1}$, Eq. (14) reduces to

$$
-\bar{A}^{\prime \prime}(\omega)+\mu^{2} \beta_{2}^{2}\left(\omega-\omega_{0}\right)^{2} \bar{A}(\omega)=\Lambda \bar{A}(\omega) .
$$

The ground-state solution of this equation is, as predicted, a Gaussian:

$$
\bar{A}(\omega)=A_{0} \exp \left[-\mu\left|\beta_{2}\right| \frac{\left(\omega-\omega_{0}\right)^{2}}{2}\right],
$$

with eigenvalue $\Lambda=\mu\left|\beta_{2}\right|$. The corresponding widths are given by the expressions

$$
\Delta t_{0}^{\mathrm{opt}}(\mu)=\sqrt{\frac{\mu\left|\beta_{2}\right|}{2}}, \quad \Delta s_{\infty}^{\mathrm{opt}}(\mu)=\sqrt{\frac{\left|\beta_{2}\right|}{2 \mu}} .
$$

Notice that in this case

$$
\Delta t_{0}^{\mathrm{opt}}(\mu) \Delta s_{\infty}^{\mathrm{opt}}(\mu)=\frac{\left|\beta_{2}\right|}{2},
$$

i.e., the product of the two spreads equals a constant.

\subsection{Waveguide dispersion}

As a second example we consider the propagation of a pulse mode in a cylindrical metallic waveguide filled with vacuum. The dispersion relation is given by [15]

$$
k(\omega)=\frac{\sqrt{\omega^{2}-\omega_{\mathrm{co}}^{2}}}{c},
$$

where $\omega_{\text {co }}$ is the waveguide's cutoff frequency. For example, for the $\mathrm{TM}_{0,1}$ mode of a waveguide with circular cross-section, 

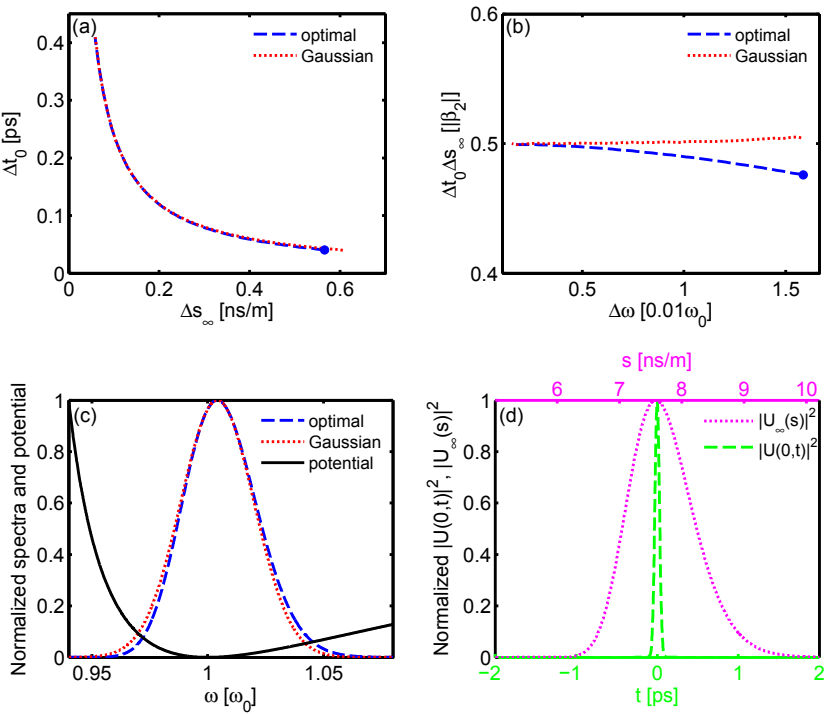

FIG. 1 Behavior of a light pulse under waveguide dispersion. (a) $\Delta t_{0}$ against $\Delta s_{\infty}$, (b) $\Delta t_{0} \Delta s_{\infty}$ as function of the spectral width, (c) the normalized optimal spectrum, the closest Gaussian spectrum and the "potential" (solid black line). In (a)-(c) the blue dashed lines and the red dotted lines correspond, respectively, to the optimal pulse and the closest Gaussian pulse. In (a) and (b) $\mu$ ranges from $7 \times 10^{-3} \mathrm{~m}$ to $7 \times 10^{-5} \mathrm{~m}$ (marked with dots). In (d) the green dashed line and the purple dotted line illustrate, respectively, the normalized pulse intensities at the input, $|U(0, t)|^{2}$, and at infinity, $\left|U_{\infty}(s)\right|^{2}$. The spectra in (c) and the lines in (d) correspond to the dots in (a) and (b). The movie shows how the various quantities evolve when $\mu$ decreases within the shown interval.

$\omega_{\mathrm{co}}=\rho_{0,1} c / r$, where $\rho_{0,1}, c$ and $r$ are, respectively, the first root of the zero-order Bessel function $J_{0}(x)$, the speed of light in vacuum, and the cylinder's radius. We choose $r=1 \mu \mathrm{m}$ leading to $\omega_{\mathrm{co}}=7.21 \times 10^{14} 1 / \mathrm{s}$, for which the wavelength is $2.61 \mu \mathrm{m}$. Furthermore, we set the pulse's central frequency close to the cutoff frequency, i.e., $\omega_{0}=1.1 \omega_{\text {co }}$ with the wavelength $2.38 \mu \mathrm{m}$.

Figure 1 illustrates the behavior of a light pulse under waveguide dispersion. In Figure 1(a) the blue dashed line, corresponding to $\Delta t_{0}^{\mathrm{opt}}$ vs. $\Delta s_{\infty}^{\mathrm{opt}}$, constitutes the boundary between the achievable and forbidden values for the two spreads of a pulse. The red dotted line corresponds to Gaussian pulses of varying width. The similarity of the curves suggests that in this case the optimal pulses are close to Gaussian. Figure 1(b) shows the product $\Delta t_{0} \Delta s_{\infty}$ for both the optimal (blue dashed line) and Gaussian (red dotted line) pulses in terms of their spectral width [calculated from Eq. (36)]. Notice that for the optimal pulses, this product falls increasingly below $\left|\beta_{2}\right| / 2$ as the spectrum widens.

Figure 1(c) shows the normalized optimal spectrum (blue dashed line), its best-fit Gaussian spectrum with the same peak frequency (red dotted line), and the "potential" given in Eq. (35) (black solid line), for $\mu=7 \times 10^{-5} \mathrm{~m}$ corresponding to the dots in Figures 1(a) and (b). Notice that the spectral peaks do not coincide exactly with the potential minimum at $\omega_{0}$, and that due to the asymmetric potential, the optimal pulse deviates slightly from Gaussian especially at the tails of the spectrum. The temporal shape of the optimal pulse at the input $\left[|U(0, t)|^{2}\right.$ from Eq. (38)], and after propagation over a long distance $\left[\left|U_{\infty}(s)\right|^{2}\right.$ from Eq. (39)] are plotted in Figure $1(\mathrm{~d})$ as

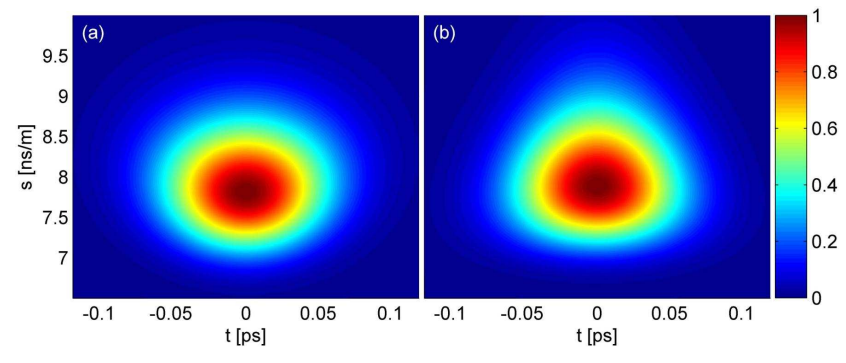

FIG. 2 Distribution of $B_{s}(0, t, s)$ for waveguide dispersion in $t, s$ space for (a) the optimal pulse, and (b) the closest Gaussian pulse.

a green dashed line and a purple dotted line, respectively, for the same $\mu$ as in Figure 1(c).

Figures 2(a) and (b) illustrate the modified Wigner function $B_{S}(0, t, s)$ for waveguide dispersion [Eq. (26) of Ref. [12] with the change of variables in Eq. (17)] in the $t, s$ space for the optimal pulse and the closest Gaussian pulse, respectively. Notice that the distribution is more compact and symmetric for the optimal pulse.

\subsection{Pure cubic dispersion}

As a third example, we consider the case when the linear part of the dispersion vanishes at a prescribed $\omega_{0}$ (the socalled zero-dispersion frequency). In practice, this is usually achieved through local cancelation of material and waveguide dispersion. The dispersion relation is then approximately

$$
k(\omega) \approx \beta_{0}+\beta_{1}\left(\omega-\omega_{0}\right)+\beta_{3} \frac{\left(\omega-\omega_{0}\right)^{3}}{6}
$$

i.e., there is no quadratic term. For fused silica, which has a zero-dispersion frequency in bulk form at $\omega_{0}=1.27 \mu \mathrm{m}$, the dispersion coefficients can be found from Sellmeier's equation [16] to be approximately $\beta_{0}=7.16 \times 10^{6} 1 / \mathrm{m}$, $\beta_{1}=4.88 \times 10^{-9} \mathrm{~s} / \mathrm{m}$, and $\beta_{3}=7.34 \times 10^{-41} \mathrm{~s}^{3} / \mathrm{m}$. Within the interval $\left[0.4 \omega_{0}, 1.6 \omega_{0}\right]$, this truncation at the cubic term only introduces a relative error in $k(\omega)$ less than $0.5 \%$.

Figure 3 illustrates the behavior of a pulse in fused silica whose spectrum is centered at the zero-dispersion frequency. The quantities in each figure are as in Figures $1(\mathrm{a})-(\mathrm{c})$, but with $\mu$ varying from $10^{-3} \mathrm{~m}$ to $10^{-5} \mathrm{~m}$ (marked with the dots) in (a) and (b). The intensity profile (not shown) becomes increasingly oscillatory on propagation. Notice that unlike in Figure 1(b), the product of the spreads in Figure 3(b) varies strongly with the spectral width. Figure 3(c) shows the spectrum of the optimal pulse and its best-fit Gaussian spectrum for the case labeled by the dots in (a) and (b). Notice the spectrum is broader than the Gaussian at the center and decays faster at the tails. This is due to the fact that the potential is quartic.

\section{CONCLUDING REMARKS}

In this work a procedure was presented for finding the shape of a pulse of given central frequency and spectral width whose temporal spread increases as slowly as possible on propaga- 

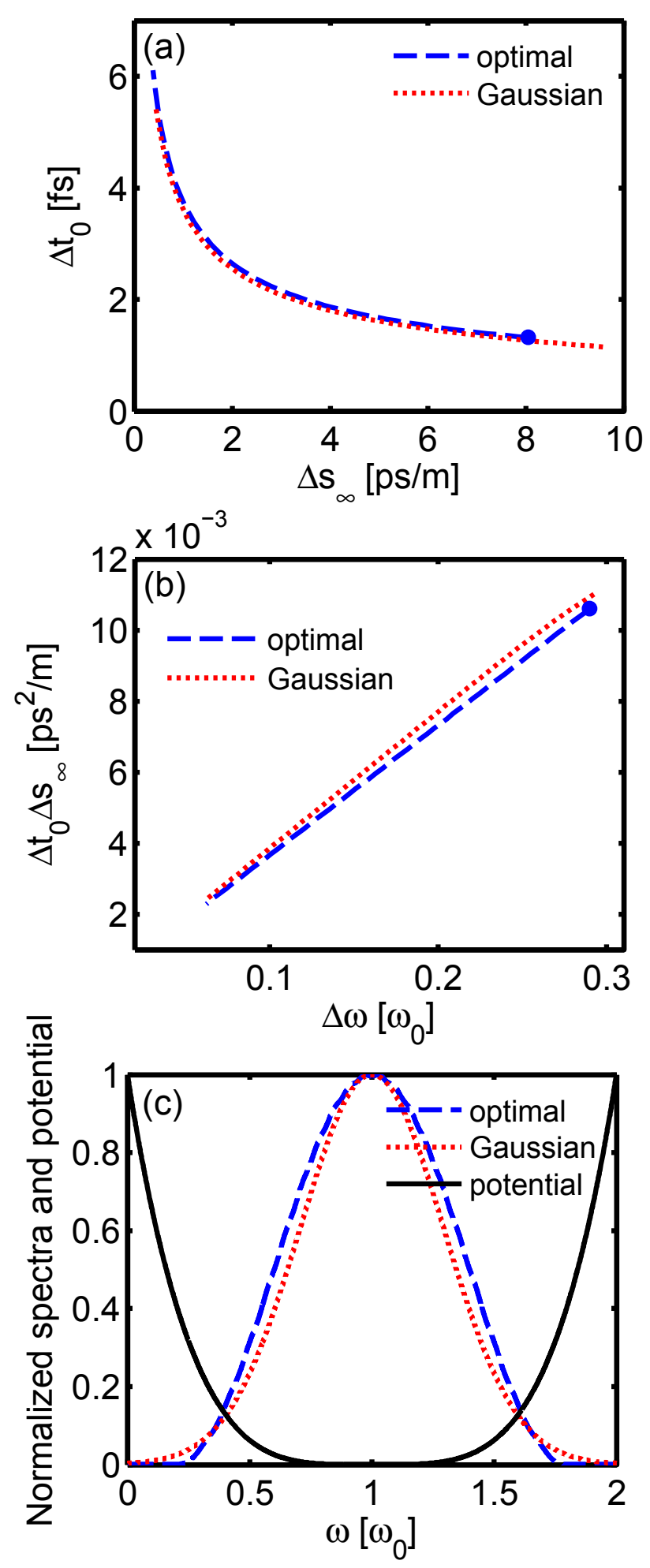

FIG. 3 Behavior of a light pulse in fused silica with the spectrum centered at the zerodispersion frequency. The quantities in the figure are the same as in Figures $1(a)-(c)$, but with $\mu$ running from $10^{-3} \mathrm{~m}$ to $10^{-5} \mathrm{~m}$ (marked with the dot) in (a) and (b). The spectra in (c) are for the minimum value of $\mu$. The movie shows how these quantities change as $\mu$ decreases.

tion in a transparent medium of arbitrary dispersion properties. This procedure was shown to reproduce the known results for the simple case of linear dispersion. It was also applied to propagation in a hollow metallic waveguide and in fused silica at the zero-dispersion frequency. For the parameters used in these examples the optimal pulses do not differ significantly from Gaussian. However, other cases will show more significant differences, for example if the central frequency is closer to the cutoff frequency of a waveguide. The optimal spectra can be generated, for example, by pulse transformations using dispersive optical elements [17]. One application of these optimal pulses could be the introduction of a pulse quality factor for propagation in specific dispersive media; such factor can be defined as the product $\Delta t_{0} \Delta s_{\infty}$ for the pulse in question, normalized by the corresponding product for the optimal pulse with the same central frequency and spectral width.

A fundamental assumption in this work was complete absence of absorption. It is known from the Kramers-Kronig relations that absorption is inherent in dispersion. Very often, absorption is negligible over the spectral regions of interest, such as in telecommunication channels. However, in cases where absorption effects must be accounted for, the procedure here could be modified accordingly: the limit $z \rightarrow \infty$ can no longer be used, but the temporal spreads at the input and at a specified finite propagation distance could be jointly minimized. Another possible generalization of the results relates to pulse trains that are temporally partially coherent.

\section{ACKNOWLEDGEMENTS}

This research was supported by the Academy of Finland (grant 128331). The work was performed when M. A. Alonso was on a sabbatical leave at the Department of Applied Physics of Aalto University in Espoo, Finland.

\section{APPENDIX A: SOLUTION OF THE EIGENVALUE PROBLEM}

Equation (14) can be solved numerically by using a HermiteGaussian expansion

$$
\bar{A}(\omega)=\sum_{n=0}^{\infty} \frac{c_{n}}{\sqrt{(\pi / a)^{(1 / 2)} 2^{n} n !}} H_{n}(\sqrt{a} \bar{\omega}) \exp \left(-a \frac{\bar{\omega}^{2}}{2}\right),
$$

where $c_{n}$ and $a>0$ are constants, $H_{n}(x)$ denotes the Hermite polynomial of order $n$, and $\bar{\omega}=\omega-\omega_{0}$. Substituting Eq. (29) into Eq. (14), and using the integral relations [18]

$$
\begin{aligned}
& \int_{-\infty}^{\infty} H_{n}(\sqrt{a} x) H_{m}(\sqrt{a} x) \exp \left(-a x^{2}\right) \mathrm{d} x=\sqrt{\frac{\pi}{a}} 2^{n} n ! \delta_{n, m}, \\
& \int_{-\infty}^{\infty} a x^{2} H_{n}(\sqrt{a} x) H_{m}(\sqrt{a} x) \exp \left(-a x^{2}\right) \mathrm{d} x \\
= & \sqrt{\frac{\pi}{a}}\left[2^{n-1}(2 n+1) n ! \delta_{n, m}\right. \\
& \left.+2^{n}(n+2) ! \delta_{n+2, m}+2^{n-2} n ! \delta_{n-2, m}\right],
\end{aligned}
$$

as well as the recurrence relations of the Hermite polynomials, we find that Eq. (14) can be written as a discrete eigenvalue problem

$$
\sum_{n=0}^{\infty} H_{m, n} c_{n}=\Lambda c_{m}
$$

where

$$
\begin{aligned}
H_{m, n} & =\frac{a}{2}(2 n+1) \delta_{n, m}-\frac{a}{2} \sqrt{(n+1)(n+2)} \delta_{n+2, m} \\
& -\frac{a}{2} \sqrt{n(n-1)} \delta_{n-2, m}+\bar{V}_{n, m},
\end{aligned}
$$


with

$$
\begin{aligned}
\bar{V}_{n, m} & =\frac{1}{\sqrt{2^{(n+m)} \pi n ! m !}} \\
& \times \int_{-\infty}^{\infty} V\left(\frac{x}{\sqrt{a}}+\omega_{0}\right) H_{n}(x) H_{m}(x) \exp \left(-x^{2}\right) \mathrm{d} x,
\end{aligned}
$$

and

$$
V(\omega)=\mu^{2}\left[k^{\prime}(\omega)-\kappa_{011}\right]^{2} .
$$

We see that the matrix $H_{m, n}$ is symmetric. In the example of waveguide dispersion, the value $a=\mu\left|\beta_{2}\left(\omega_{0}\right)\right|$ is used for the width of Gaussian functions, as suggested by the analytical solution in the case of linear dispersion, Eq. (24). For the zero-dispersion case, we use instead the expression $a=70\left[\mu\left|\beta_{3}\left(\omega_{0}\right)\right|\right]^{2 / 3}$, since $\beta_{2}=0$ at the zero-dispersion frequency.

Once $\bar{A}(\omega)$ is found, the spectral amplitude is given by $A(\omega)=\bar{A}(\omega) \exp \left(i \kappa_{100} \omega\right)$. The spectral width can be estimated as

$$
\Delta \omega=\sqrt{\left(\overline{\omega^{2}}\right)-\left(\overline{\omega^{1}}\right)^{2}}
$$

where

$$
\left(\overline{\omega^{m}}\right)=\frac{\int \omega^{m}|A(\omega)|^{2} \mathrm{~d} \omega}{\int|A(\omega)|^{2} \mathrm{~d} \omega}, \quad m=(1,2),
$$

and the integration extends over the range in which the spectrum is nonzero.

The initial pulse in the time domain is obtained by setting $z=0$ in Eq. (1), and then (inverse) Fourier transforming the spectrum, given by Eq. (29). The result is

$$
\begin{aligned}
U(0, t)= & \exp \left[i \omega_{0}\left(\kappa_{100}-t\right)\right] \\
& \times \sum_{n=0}^{\infty} \frac{(-i)^{n} c_{n}}{\sqrt{(a \pi)^{1 / 2} 2^{n} n !}} H_{n}\left(\frac{\kappa_{100}-t}{\sqrt{a}}\right) \\
& \times \exp \left[-\left(\kappa_{100}-t\right)^{2} / 2 a\right] .
\end{aligned}
$$

Since only the pulse envelope is of interest, we set $\kappa_{100}=0$ leading to $|U(0, t)|$ centered at $t=0$.

The pulse at an infinite distance in terms of $s$ is obtained from Eq. (1) by inserting $t=s z$, taking the limit $z \rightarrow \infty$, and carrying out the integration using the method of stationary phase. Doing so, one finds that

$$
\begin{aligned}
U_{\infty}(s)= & \lim _{z \rightarrow \infty} U(z, s z), \\
= & \frac{A\left(\omega_{s}\right)}{\sqrt{z\left|\Omega^{\prime \prime}\left(\omega_{s}\right)\right|}} \exp \left[i z \Omega\left(\omega_{s}\right)\right] \\
& \times \exp \left\{i \pi \operatorname{sign}\left[\Omega^{\prime \prime}\left(\omega_{s}\right)\right] / 4\right\},
\end{aligned}
$$

where $\omega_{s}$ is determined by $k^{\prime}\left(\omega_{s}\right)=s, \Omega(\omega)=k(\omega)-\omega s$, and $\operatorname{sign}(x)$ equals 1 for $x>0$ and -1 for $x<0$.

\section{References}

[1] A. E. Siegman, "New developments in laser resonators" Proc. SPIE 224, 2-14 (1990).

[2] P. A. Bélanger, Y. Champagne, and C. Paré, "Beam propagation factor of diffracted laser beams" 0pt. Commun. 105, 233-242 (1994).
[3] P. A. Bélanger, "Beam propagation and the ABCD ray matrices" Opt. Lett. 16, 196-198 (1991).

[4] A. E. Siegman, "Defining the effective radius of curvature for a nonideal optical beam" IEEE J. Quantum Elect. 27, 1146-1148 (1991).

[5] H. Weber, ed., Special Issue on Laser Beam Quality, Opt. Quant. Electron. 24, no. 9 (1992).

[6] S. A. Ponomarenko, and G. P. Agrawal, "Phase-space quality factor for ultrashort pulsed beams" Opt. Lett. 3, 767-769 (1989).

[7] B. H. Kolner, and M. Nazarathy, “Temporal imaging with a time lens" Opt. Lett. 14, 630-632 (1989).

[8] B. H. Kolner, "Space-time duality and the theory of temporal imaging" IEEE J. Quantum Elect. 30, 1951-1963 (1994).

[9] B. E. A. Saleh, and M. C. Teich, Fundamentals of Photonics (Second Edition, Wiley, New York, 2007).

[10] G.-C. Lin, C.-H. Sui, and Q. Lin, “Non-Gaussian pulse propagation and pulse quality factor using intensity moment method" Chinese Phys. Lett. 16, 415-417 (1999).

[11] G. Rousseau, N. Mcgarthy, and M. Piché, "Description of pulse propagation in a dispersive medium by use of a pulse quality factor," Opt. Lett. 27, 1649-1651 (2002).

[12] J. C. Petruccelli and M. A. Alonso, "Phase space distribution tailored for dispersive media," J. Opt. Soc. Am. A 27, 1194-1201 (2010).

[13] P. Loughlin, and L. Cohen, “A Wigner approximation method for wave propagation" J. Acoust. Soc. Am. 118, 1268-1271 (2005).

[14] P. Loughlin, and L. Cohen, "Approximate wave function from approximate non-representable Wigner functions" J. Mod. Opt. 55, 3379-3387 (2008).

[15] J. Vanderlinde, Classical Electromagnetic Theory (Wiley, New York, 1993).

[16] G. P. Agrawal, Nonlinear Fiber Optics (Second Edition, Academic Press, San Diego, 1995).

[17] N. G. R. Broderick, "Method for pulse transformations using dispersion varying optical fibre tapers" Opt. Express 18, 24060-24069 (2010).

[18] G. B. Arfken, and H. J. Weber, Mathematical Methods for Physicists (Sixth Edition, Elsevier, Amsterdam, 2005). 